\title{
Surface complexation models for heterogeneous and uneven surfaces - the charge regulation concept applied to simple 2D geometries
}

\author{
FRANK HEBERLING ${ }^{1}$, TEBA GIL-DÍAZ ${ }^{2}$ AND \\ JOHANNES LUETZENKIRCHEN ${ }^{3}$ \\ ${ }^{1}$ Karlsruhe Institute of Technology (KIT) \\ ${ }^{2}$ Karlsruhe Institute of Technology \\ Presenting Author: frank.heberling@kit.edu
}

${ }^{3} \mathrm{KIT}$

Electrostatic surface complexation models ( $\mathrm{SCMs}$ ) are widely used to describe the adsorption of ions and the charging behavior at mineral surfaces. Classical SCMs have been developed to describe simple particle geometries (flat, spherical, cylindrical). The models imply that the electrostatic potential, developing at charged mineral-water interfaces, approaches zero in the bulk solution and is not laterally influenced by other potentials [1].

At the pore-scale, especially in nano-porous systems, but also in pore throats of larger pores or at patch-wise heterogeneous surfaces, such an assumption is not valid. Potentials originating from adjacent surfaces overlap. The charge regulation approach accounts for this overlap in electrostatic potentials and its impact on the speciation of the involved surfaces. Charge regulation mitigates between the simplifying constant potential and constant charge approaches and has been used very successfully to interpret force distance data as obtained with atomic force microscopes or force distant apparatus. Recently, a general purpose code was developed to simulate 1D charge regulated systems for arbitrary surface chemistries allowing the use of all variants of SCM in arbitrary electrolyte solutions [2].

In this contribution we present a next step in our code development. The 2D Poisson-Boltzmann equation for arbitrary electrolytes is solved by a Finite-Element method (FEnics, Python), coupled to arbitrary SCMs that may for example represent nano-scale sections of a patch-wise surface. Selected examples will demonstrate the effects of electrostatic potential overlap on flat heterogeneous surfaces, or on uneven surfaces (represented by corners/edges). The results of the corner simulations demonstrate that chemical heterogeneity can be generated on a surface, which is homogeneous concerning the distribution of functional groups, due to geometry and overlapping electrostatic potentials. The results provide unprecedented insight into pore-scale reactivity and may have important implications for modelling adsorption reactions in nano-porous systems or at pore-throats.

We acknowledge funding from the German Federal Ministry of Education and Research (02NUK053C) and the Helmholtz association (SO-093).

[1] Lützenkirchen, J., Surface complexation modelling. Academic press: 2006; Vol. 11.

[2] Gil-Díaz, T., et al., Charge regulated solid-liquid interfaces interacting on the nanoscale: benchmarking of a generalized speciation code, Advances in Colloid and Interface Science (submitted) 